\title{
La dirección de Trabajos Fin de Grado: Una perspectiva docente de buenas prácticas
}

\section{The supervision of undergraduate students' dissertations: a perspective from the supervisors}

\author{
Fernando Lera-López ${ }^{1}$
}

Fecha de recepción: 18/02/2021; Fecha de revisión: 13/08/2021; Fecha de aceptación: 05/20/2021

Cómo citar este artículo:

Lera-López, F. (2021). La dirección de Trabajos Fin de Grado: Una perspectiva docente de buenas prácticas. Revista de Innovación y Buenas Prácticas Docentes, 10(2), 117-130.

Autor de Correspondencia: lera@unavarra.es

\section{Resumen:}

La realización del Trabajo Fin de Grado (TFG) constituye para el alumno universitario su primer trabajo de investigación que debe realizar de manera autónoma, con la supervisión de un docente. Distintos trabajos a nivel internacional han analizado esta relación entre alumno y docente, principalmente considerando la perspectiva del primero. Este trabajo constituye una novedad para el análisis de la supervisión de TFG en España, analizando la perspectiva del docente universitario para buenas prácticas docentes. Para ello, se han manejado dos encuestas a 29 docentes pertenecientes a 9 universidades públicas españolas, que han valorado esta relación y el rol desempeñado tanto por el docente como por el alumno. Los resultados obtenidos muestran que el uso de los medios digitales es muy importante en las relaciones de comunicación y supervisión de los TFG. En cuanto al propio docente, se debe motivar y animar al alumno, además de comprometerse a responderle en un plazo determinado. Respecto a los estudiantes, el estudio refleja una serie de deficiencias específicas, como es la redacción del trabajo, junto con otras más genéricas de compromiso y autonomía. Estos resultados pueden ofrecer orientaciones e indicaciones al docente universitario de cara a una mejor dirección de los trabajos fin de grado.

Palabras clave: estudios universitarios, aprendizaje; supervisión; tecnologías de la información y la comunicación.

\begin{abstract}
:
Different studies at international level have analyzed the relationship between student and supervisor in the development of the undergraduate dissertation. Nevertheless, studies have mainly considered the students' perspective. This paper is a novelty for the analysis in the supervision of undergraduate dissertations in Spain, analyzing the supervisor's approach. For this, two surveys have been conducted to 29 teachers with experience in undergraduate dissertation supervision belonging to 9 Spanish public universities, who have valued the relationship between supervisors and students. Likewise, the use of digital media for the development of this supervision has been considered. The results show that the use of digital media is very important in communication relationship and supervision of the undergraduate dissertation. According to the survey, supervisor should mainly motivate and encourage students, in addition to committing to respond within a certain period. Regarding students, the study reflects a series of specific deficiencies, such as the writing of the dissertation along with other more general commitments and autonomy, associated with their ignorance of what the realization of it entails. These results can offer orientations and indications to the supervisors towards a better supervision of the undergraduate dissertation.
\end{abstract}

Key Words: university studies; learning; supervision; information and telecommunication technologies.

\footnotetext{
I Universidad de Navarra (España), lera@unavarra.es
} 


\section{INTRODUCCIÓN}

La realización del Trabajo Fin de Grado (TFG, en adelante) constituye para muchos alumnos la realización de su primer trabajo de investigación autónomo, donde deben ofrecer evidencia del logro de una serie de competencias y habilidades. Como señalan Hannigan y Burnard (2001), el TFG permite testar una serie de importantes habilidades, como la capacidad de establecer una pregunta de investigación en un campo científico, la capacidad para obtener la literatura relevante y estructurar adecuadamente un trabajo, así como de relacionar teoría y práctica en un determinado campo de conocimiento. Justamente, la necesidad de que el alumno lo realice con alto grado de autonomía, hace que resulte esencial en su desarrollo la relación entre el alumno y el tutor (Greenbank \& Penketh, 2009; Hannigan \& Burnard, 2001; Mills \& Matthews, 2009), lo que convierte también en complejo el equilibrio entre autonomía del alumno y apoyo del tutor (Del Río, Díaz-Vázquez, \& Maside Sanfiz, 2018). En el marco de esa relación, el alumnado puede no solo demostrar las competencias que ha ido adquiriendo, aplicándolas a una materia específica, sino también incrementar su autoestima y autonomía (Todd, Bannister, y Clegg, 2004), junto con las habilidades y competencias que le van a ser de utilidad en su incorporación al mercado laboral (Feather, Ancho, \& Cowton, 2014).

A pesar de la importancia académica que tiene la realización del TFG, hay escasa evidencia empírica acerca del proceso de dirección de los TFG, centrándose más en el ámbito de la evaluación de estos (Ortiz-Repiso, García Zorita, Pacios, \& Vianello, 2014; Starr-Glass \& Ali, 2012; Webster, Pepper, \& Jenkins, 2000) que en el proceso de gestión y dirección de aquellos (Derounian, 2011; Expósito et al., 2018; Rowley \& Slack, 2004; Shadforth \& Harvey, 2003). No obstante, un elemento esencial del éxito en la realización del TFG es la labor y el papel ejercido por el tutor, apoyando y guiando al alumno (Expósito Díaz et al., 2018). Conviene señalar además que la dirección de TFG puede ser una tarea intensiva en tiempo para el tutor, y donde el rol que ejerce el tutor puede variar en el tiempo, desarrollándose distintos roles complementarios (Rowley \& Slack, 2004).

En este contexto, la escasa evidencia empírica se ha centrado más en la perspectiva del alumno que del docente, analizando las peculiaridades de otros sistemas universitarios extranjeros, especialmente en el ámbito anglosajón. En el panorama español, los estudios son muy escasos, y no abordan la valoración de las percepciones de los tutores de los TFG. En este sentido, el presente trabajo pretende ofrecer una cierta panorámica de estas a partir de la opinión de un grupo de 29 tutores de distintas universidades públicas españolas. Los resultados aquí obtenidos pueden ayudar a reflexionar sobre esta supervisión y mejorarla en el futuro.

\section{LA DIRECCION DE TRABAJOS FIN DE GRADO}

Con la implantación del Plan Bolonia y la aparición de los nuevos grados universitarios en España, el TFG aparece como una asignatura más que se debe realizar en el último curso. Su marco legal se detalla en el Real Decreto 1393/2007, y posterior modificación por Real Decreto 861/2010, donde se establece un intervalo de créditos muy amplio, que va de 6 a 30 créditos. Asimismo, se indica que el TFG está orientado a la evaluación de las competencias asociadas a la titulación que ha cursado el alumno. De este modo, el marco legal deja en manos de cada universidad la regulación del proceso de elaboración de este y el método de evaluación, según las características que presente el correspondiente grado. Esta flexibilidad y autonomía que van a tener las universidades en la regulación de los TFG se manifiesta no solo en diferencias entre las distintas universidades, sino también en diferencias notorias entre Escuelas y Facultades de una misma universidad. 
Asimismo, nos encontramos con al menos cuatro tipos de TFG: experimentales, de revisión e investigación bibliográfica, de carácter teórico-práctico y de carácter profesional, asociados fundamentalmente al desarrollo de prácticas empresariales (Rekalde-Rodríguez, 2011).

Tres son los elementos más importantes que el alumno debe de desarrollar en su TFG. En primer lugar, el desarrollo de su autonomía. El TFG supone la elaboración de un trabajo de manera autónoma y guiado por un tutor. Esto implica para el alumno el desarrollo de su iniciativa personal, así como poner en marcha competencias que a lo largo del grado ha podido ir desarrollando, aunque de manera fragmentada en distintas asignaturas. Una labor importante del tutor en el fomento de la autonomía del alumno es dotarle de los apoyos adecuados para que lo pueda realizar por sí mismo, siendo consciente que para el alumno es la primera vez que se enfrenta a un trabajo científico de esta envergadura. En segundo lugar, la profesionalidad del alumno asociada a la realización de un trabajo de carácter científico. La inclusión del TFG en los planes de estudios universitarios obedece a que la realización de este dotará a los alumnos de las habilidades necesarias para fundamentar su práctica profesional futura. Algunos autores han enfatizado que "la clave del TFG no radica en los resultados de investigación, sino en cómo se ha llegado a ellos movilizando competencias claves del grado" (RekaldeRodríguez, 2011, p.190). En tercer lugar, la creatividad que tiene que desarrollar el alumno para abordar con éxito el TFG. El TFG permite al estudiante desplegar y desarrollar muchos de los aspectos más importantes que ha ido aprendiendo a lo largo de 3-4 años desde un punto de vista personal. Esa creatividad que exige el TFG se manifiesta en que debe de ser el alumno el que debe acotar, con el apoyo del tutor, el tema objeto de estudio. Esto lógicamente afectará positivamente a su motivación, dado que su implicación en el desarrollo del TFG debe de ser total. Belletich Ruíz y Pérez de Villarreal (2016) analizan los principales obstáculos que encuentran los mismos, destacando el desconocimiento sobre lo que es un TFG, además de las dificultades para realizar labores de análisis y síntesis, así como de consulta de fuentes.

Las diferencias interuniversitarias e intrauniversitarias en el desarrollo de los TFG, previamente señaladas, se manifiestan claramente en el papel que debe de ejercer el tutor en el desarrollo del TFG, con distintas percepciones de este por parte de los estudiosos, como indica Heinze y Heinze (2009). Siguiendo a Todd et al. (2004), cuatro son las grandes funciones que debe de desarrollar el tutor en la dirección del TFG. En primer lugar, debe proveer de apoyo y guía académico, facilitando información, recursos y metodología para desarrollar el TFG. En segundo lugar, deber ser un dinamizador y facilitador del proceso de aprendizaje del alumno, motivando a este. En tercer lugar, debe de ofrecer un conocimiento asociado al objeto de investigación del TFG. Finalmente, debe ayudar en la redacción del TFG, siendo este el aspecto más controvertido (Del Río et al., 2018; Romero-Oliva, 2020, Sáez, 2017; Todd, Smith, \& Bannister, 2006). En este contexto, algunas universidades han planteado la necesidad de dar apoyo a la labor de tutorización del docente con la realización de cursos de formación al alumnado sobre técnicas de escritura, de búsqueda de información, etc. 
Hay autores que señalan que el rol ejercido por el tutor influye en las habilidades desarrolladas por el alumno en su TFG (Expósito et al., 2018; Del Río et al., 2018). Por ello, resulta imprescindible el desarrollo de un correcto proceso de supervisión del TFG. Distintos trabajos han abordado a partir de las percepciones y experiencias de alumnos este proceso de relación entre alumno y tutor (ej., Augustsson, \& Jaldemark, 2014; Belletich Ruíz \& Pérez de Villarreal, 2016; Del Río et al., 2018; Feather et al., 2014; Greenbank \& Penketh, 2009; Heinze \& Heinze, 2009; Jaldemark \& Lindberg, 2013; Malcolm, 2012; Mills \& Matthews, 2009; Todd et al., 2004; Vera y Briones, 2015). Sin embargo, en el caso de los tutores, la evidencia es más escasa (ej., Derounian, 2011; Feather et al., 2014; Malcolm, 2012; Mills \& Matthews, 2009; Roberts \& Seaman, 2018), sin que se hayan encontrado muchos trabajos en el ámbito español (Expósito et al., 2018).

La naturaleza de esta relación va variando en el tiempo, según el alumno va ganando en seguridad y autonomía en el desarrollo del TFG, reduciendo la necesidad de reuniones. Asimismo, las necesidades específicas de cada alumno van a condicionar estas relaciones, para conjugar un equilibrio adecuado entre autonomía y supervisión, que evite que el alumno pueda sentirse frustrado o confundido en el desarrollo de su TFG, por una posible carencia de autonomía (Rowley \& Slack, 2004). Así, distintas evidencias empíricas abogan por una relación en la que prime el diálogo más que la transmisión de ideas, y donde el tutor plantee más preguntas que respuestas, de modo que sea el propio alumno el que tenga que tomar decisiones, y no sea el tutor el que imponga su opinión, creando alumnos conformistas y dependientes (Greenbank \& Penketh, 2009). Otros autores insisten en la importancia de establecer una reglas claras de funcionamiento, especificando cuándo y cómo reunirse, los documentos a revisar, los resultados de cada reunión, etc. (Hannigan \& Burnard, 2001). Otros trabajos enfatizan el aspecto relacional, destacando la necesidad del consejo claro y directo del tutor, y su apoyo y motivación constante al alumno (Roberts \& Seaman, 2018). Para mejorar este proceso de supervisión del tutor, distintos autores han propuesto el uso de distintas estrategias de retroalimentación, así como tutorías grupales para abordar cuestiones generales (Mills \& Matthews, 2009).

Por otro lado, el desarrollo de las tecnologías de la información y la comunicación, y su aplicación al ámbito educativo ha permitido la aparición de la supervisión online, mediante la cual la comunicación escrita y oral se realiza a través de ordenadores, inicialmente, y con el uso de móviles y tablets posteriormente. Lógicamente, esta manera de supervisión no está exenta de problemas, que distintos trabajos han ido analizando (Augustsson \& Jaldemark, 2014; Heinze \& Heinze, 2009; Sussex, 2008).

Hannigan y Burnard (2001) han definido las principales etapas en la realización del TFG y el rol a ejercer por el tutor que, aunque aplicado al campo de la enfermería, pueden ser generalizadas a cualquier TFG. Estos autores insisten en la importancia de que el alumno tenga claro qué es lo que se exige en un TFG, la elección de una pregunta de investigación clara y concreta, y la capacidad de establecer una correcta planificación del trabajo a desarrollar. La selección de las referencias bibliográficas relevantes y una buena síntesis de lo que aportan en el contexto de la pregunta de investigación es muy importante, y permite también el logro de competencias digitales informacionales (Rowley 6 Slack, 2004).

Asimismo, distintos trabajos insisten en la importancia de desarrollar una estructura lógica y clara en el TFG, además de que esté correctamente escrito. Por ejemplo, Greetham (2009) enfatiza la importancia de escribir correctamente, señalando los principales problemas de redacción. Para facilitar este proceso, se han establecido herramientas específicas para el seguimiento y evaluación de los TFG en determinadas titulaciones (Ortiz-Repiso et al., 2014). 


\section{DESARROLLO DE LA EXPERIENCIA DE INNOVACIÓN}

\subsection{Diseño}

El presente trabajo pretende reflexionar sobre las percepciones que tienen los tutores de los TFG tanto de sí mismos como de sus alumnos, de tal modo que el proceso de dirección de TFG pueda mejorar en el tiempo. Distintos trabajos en países europeos han analizado con muestras relativamente reducidas (tamaño inferior a 20 tutores), dichas percepciones (ej., Derounian, 2011; Malcolm, 2012; Mills \& Matthews, 2009), pero en castellano la evidencia es menor (Expósito et al., 2018). Con este trabajo se pretenden estudiar las distintas etapas en la realización del TFG y el rol que debe asumir en cada una de ellas el alumno y el profesor, así como destacar el uso de los medios online en la supervisión del TFG. Todo ello permitirá en un futuro el diseño de buenas prácticas en la tutorización de estos.

Para el logro de estos objetivos se han desarrollado dos trabajos de campo que han permitido recopilar información de carácter tanto cualitativo como cuantitativo a partir de la información suministrada por profesores universitarios de nueve diferentes universidades españolas con experiencia en la dirección de TFG. Para ello, se ha aplicado un cuestionario dividido en tres partes: (i) una primera parte de preguntas clasificatorias con el que encuadrar el perfil del tutor de un TFG; (ii) una segunda parte dirigida a conocer la experiencia como director de un TFG, con especial énfasis en identificar el grado de adecuación de los tutores a los medios online; y (iii) una tercera parte con el objetivo de descubrir las características más importantes que debe de tener un tutor en la dirección de los TFG. El cuestionario aplicado se ha basado en trabajos previos de encuestas a tutores, como los elaborados por Derounian (2011) y Hannigan y Burnard (2001), entre otros. Asimismo, la importancia dada en trabajos previos al uso de los medios online en la supervisión de TFG (Heinze y Heinze, 2009; Jaldemark y Lindberg, 2013), nos ha llevado a incluir preguntas sobre el papel de los mismos en dicha supervisión. El cuestionario fue validado previamente con la realización de un pretest entre profesores de la Universidad Pública de Navarra que han dirigido TFG.

\subsection{Muestra y análisis de datos}

Partiendo de dos encuestas que se realizaron en los cursos 2018-19 (Campus Iberus) y 2019-20 (Campus Virtual Compartido G9) a profesores universitarios que han dirigido TFG, se han obtenido los resultados estadísticos que aquí se presentan. La primera encuesta se realizó para profesores del Campus Iberus (Universidad de Zaragoza, Universidad Pública de Navarra, Universidad de La Rioja y Universidad de Lleida), mientras que la segunda incluía a profesores del Campus Virtual Compartido G9, que engloba a las siguientes universidades: Universidad de Zaragoza, Universidad Pública de Navarra, Universidad de La Rioja, Universidad de Oviedo, Universidad del País Vasco, Universidad de las Islas Baleares, Universidad de Castilla-La Mancha, Universidad de Cantabria, y Universidad de Extremadura. El criterio de selección de los docentes encuestados fue su participación en dos cursos sobre la dirección de TFG impartidos por el investigador.

En concreto, la encuesta se distribuyó a través de una plataforma virtual, y los encuestados, todos ellos participantes en ambos cursos, remitieron sus respuestas por medios electrónicos. El conjunto de la población de estudio ha sido el total de profesores 
participantes en los dos cursos. En cuanto a la muestra con la se ha contado, un total de 29 profesores contestaron válidamente a la encuesta. Las principales características generales de los profesores encuestados son las siguientes: los tutores tienen en término medio una edad de 43 años, llevando 13 años a tiempo completo en la universidad y 1,6 años a tiempo parcial. De este modo, se puede observar que se trata de una muestra de profesores con una amplia experiencia docente universitaria.

En cuanto al ámbito de la dirección de TFG, la experiencia media como tutor es de 4 cursos, en los que dirigen de media 2 TFG al año. El 79,31\% de los encuestados ha sido miembro de tribunal de evaluación, contándoles 9,5 horas por curso en el Plan de Ordenación Docente. El promedio de créditos ECTS correspondientes a un TFG asciende a 11 créditos. Los sistemas de valoración del TFG se dividen de tal manera que, el $27,30 \%$ del peso de la nota corresponde a la valoración emitida por el tutor, el $36,25 \%$ corresponde al trabajo escrito y el $36,45 \%$ a la presentación oral de los resultados por parte del alumno, evaluados estos dos últimos aspectos por un tribunal.

Finalmente, los docentes dedican una media de 3,6 horas a la semana a supervisión de trabajos, realizando al semestre alrededor de 8 reuniones físicas con el alumno, dentro de los intervalos manejados por otros trabajos internacionales (Heinze \& Heinze, 2009; Mills \& Matthews, 2009). En cuanto al sistema de elección de los alumnos del tema y el tutor, en el $13,04 \%$ de las ocasiones el profesor selecciona al alumno, llegando a un acuerdo para el tema, mientras que en el 78,26 \% el profesor ofrece un tema, y posteriormente el alumno elige el tema que más le gusta, y por tanto al tutor. En el resto de los casos el alumno contacta con un tutor específico para el desarrollo de un tema concreto. En la tabla 1 se resumen las características clasificatorias más importantes de los tutores encuestados.

Tabla 1.

Estadística descriptiva.

\begin{tabular}{lcc}
\hline \multicolumn{1}{c}{ Variable } & Media & Desviación típica \\
\hline Edad & 43 & 9,07 \\
\hline Años en la universidad a tiempo completo & 13 & 9,08 \\
\hline Años en la Universidad a tiempo parcial & 1,60 & 2,94 \\
\hline Cursos académicos como tutor & 4,00 & 4,84 \\
\hline № de TFG supervisados al año & 2,80 & 2,85 \\
\hline Haber sido miembro del tribunal de TFG & $79,31 \%$ & --- \\
\hline Fuente: Elaboración propia & &
\end{tabular}

\section{RESULTADOS}

El análisis de los resultados obtenidos a partir de las encuestas ha sido dividido en tres partes: (i) una primera parte dirigida a conocer las opiniones de los tutores acerca de las principales dificultades que encuentran los alumnos; (ii) una segunda parte con el objetivo de descubrir las características más importantes que debe de tener un tutor en la dirección de los TFG; y (iii) finalmente un análisis del uso de medios online en la supervisión del TFG.

\subsection{Percepciones acerca de las dificultades de los alumnos para desarrollar el TFG}

Según los profesores encuestados, se muestra de modo general que los alumnos tienen claros los aspectos formales relacionados con el TFG, como son los plazos y requerimientos y el modo de evaluación. No obstante, parecen tener menos claro qué es un TFG y especialmente cómo se hace, lo cual puede sugerir la necesidad de explicar ante al alumno en qué consiste este por parte de las Facultades, Centros y Escuelas de la universidad. 
En cuanto a las características que más valoran los docentes respecto a los alumnos, tal y como se detalla en la tabla 2 , se valora con mayor puntuación la relación mantenida con ellos durante la realización del trabajo. Por el contrario, reciben puntuaciones menores el nivel de competencias del alumno, su grado de autonomía y el compromiso y dedicación del mismo. Todo este análisis nos debería llevar, con la cautela necesaria debido al tamaño muestral, a insistir en la mejora del proceso formativo del alumno en sus estudios universitarios para el desarrollo de determinadas competencias, además del fomento de la autonomía y compromiso del alumno en la realización del TFG, pero también sin olvidar que en otras asignaturas del grado se puede ir fomentando dicha autonomía en trabajos de menor envergadura.

Finalmente, en ámbito de las principales dificultades, destaca por encima de todas, la redacción del TFG. Esta situación justificaría, como se ha comentado en la introducción, que algunas universidades desarrollen talleres de escritura. Por otro lado, no dejar de ser ciertamente sorprendente que, tras cursar cuatro años de estudios universitarios, la expresión escrita constituya la principal dificultad según los tutores para la elaboración de un TFG. Distintos trabajos se han dedicado a abordar estos problemas de redacción (Da Cunha, 2016; Romero-Oliva, 2020).

Tabla 2.

El alumno desde el punto de vista del docente.

\begin{tabular}{|c|c|c|}
\hline Variable & Media & $\begin{array}{l}\text { Desviación } \\
\text { típica }\end{array}$ \\
\hline \multicolumn{3}{|l|}{ Alumno tiene claro... (del 1 al 5) } \\
\hline Qué es un TGF y qué exige & 2,62 & 1,17 \\
\hline Cómo hacer un TFG & 2,12 & 0,85 \\
\hline Que el tutor es un guía (1 al 5) & 3,41 & 1,17 \\
\hline Los plazos y requerimientos formales & 3,54 & 1,17 \\
\hline Cómo se le va a evaluar el TFG & 3,37 & 1,24 \\
\hline \multicolumn{3}{|c|}{ Valoración de los siguientes aspectos (del 1 al 10) } \\
\hline Grado de autonomía del alumno & 6,18 & 1,65 \\
\hline Nivel de compromiso y dedicación & 6,18 & 1,65 \\
\hline $\begin{array}{l}\text { Competencias del alumno para el desarrollo } \\
\text { del TFG }\end{array}$ & 6,00 & 1,51 \\
\hline $\begin{array}{l}\text { Valoración de la relación mantenida con el } \\
\text { alumno }\end{array}$ & 7,18 & 1,36 \\
\hline $\begin{array}{l}\text { Grado de satisfacción que produce la } \\
\text { realización del TFG }\end{array}$ & 7,09 & 1,90 \\
\hline \multicolumn{3}{|c|}{$\begin{array}{l}\text { Importancia del papel del tutor en las siguientes etapas } \\
\text { (del } 1 \text { al 10) }\end{array}$} \\
\hline Estableciendo el tema & 7,66 & 2,47 \\
\hline Revisión de la literatura & 6,45 & 2,22 \\
\hline Selección de la metodología apropiada & 7,45 & 1,81 \\
\hline El análisis de datos & 6,45 & 1,88 \\
\hline Revisión de los capítulos del TFG & 7,71 & 1,85 \\
\hline Nota final del TFG & 6,42 & 2,51 \\
\hline \multicolumn{3}{|c|}{$\begin{array}{l}\text { Valoración de la dificultad para el alumno las } \\
\text { siguientes etapas (1 dificultad mínima, } 5 \text { máxima): }\end{array}$} \\
\hline Establecer el tema & 3,68 & 1,81 \\
\hline Revisión de la literatura & 3,58 & 2,10 \\
\hline Selección de la metodología & 3,68 & 1,97 \\
\hline Análisis de los datos & 3,87 & 2,11 \\
\hline Redacción del TFG & 4,33 & 2,05 \\
\hline Presentación del TFG & 3,83 & 2,35 \\
\hline
\end{tabular}




\subsection{Importancia del tutor en la supervisión del TFG}

Resulta muy interesante analizar la importancia del docente como tutor en las distintas etapas de desarrollo del TFG. El establecimiento de las distintas etapas se ha realizado a partir de la revisión de la literatura (ej., Hannigan \& Burnard, 2001). De este modo, las etapas en las que función del docente parece más relevante son tres: revisión del TFG, establecimiento del tema y selección de la metodología. Distintos trabajos empíricos confirman este resultado (Expósito et al., 2018; Malcolm, 2012; Rowley \& Slack, 2004), tanto en lo que se refiere a la elección del tema (Cook, 1980; Derounian, 2011), la selección de la metodología (Derounian, 2011; Todd et al., 2006), como la revisión de los progresos del estudiante en la redacción del TFG (Derounian, 2011). Asimismo, se observa que los docentes que supervisan más TFG destacan especialmente su papel como tutores en la selección de la metodología y en el análisis de datos.

Estas consideraciones están asociadas a las dificultades previas señaladas en el apartado anterior, en términos de las competencias adquiridas con anterioridad por los estudiantes, que a raíz de este resultado pueden estar orientadas en problemas metodológicos y en dificultades para escribir de manera correcta. Asimismo, las carencias en términos de autonomía, detectadas en la Tabla 2, justificarían una mayor importancia del tutor en la selección del tema, y las fases de diseño posterior, como destaca Expósito et al. (2018) para una muestra entre tutores. Esta etapa es juzgada también como fundamental por otros trabajos empíricos, dado que va a marcar las etapas posteriores y constituye un elemento importante de automotivación para el alumno (Derounian, 2011; Malcolm, 2012). Por otro lado, en las tareas más mecánicas o formalmente más establecidas, como son el proceso de revisión de la literatura y el análisis de los datos, la importancia del tutor es menor. Sin embargo, desde la perspectiva del estudiante, estas actividades del tutor son importantes (Del Río et al., 2018).

En opinión de los encuestados en cuanto a las distintas funciones que debe desarrollar el tutor, según se detalla en la tabla 3, los docentes valoran en un alto grado que el tutor debe motivar y animar al alumno, además de comprometerse a responder en un plazo determinado a las cuestiones del alumno, confirmando resultados previos (Jaldemark \& Lindberg, 2013; Roberts \& Seaman, 2018). En el otro extremo, los docentes encuestados señalan que el papel del tutor no debe ser el de ayudar a escribir el TFG, ni ser un mero revisor del TFG (Expósito et al., 2018), ni debe de seleccionar el tema del TFG o los objetivos específicos del mismo. De este modo, se pone de manifiesto, de nuevo, la importancia de la autonomía del alumno, por parte de los docentes, especialmente en el tema objeto de análisis, que debe de proceder de las inquietudes intelectuales que tenga el mismo, ya que ello le servirá de aliciente y motivación en el desarrollo del TFG.

Tabla 3.

Valoración del docente sobre la labor del tutor en la supervisión del TFG.

\begin{tabular}{lcc}
\hline \multicolumn{1}{c}{ Variable } & Media & $\begin{array}{c}\text { Desviación } \\
\text { típica }\end{array}$ \\
\hline Importancia del tutor en las siguientes etapas (1 al 10) & \\
\hline Estableciendo el tema & 7,66 & 2,47 \\
\hline Revisión de la literatura & 6,45 & 2,22 \\
\hline Selección de la metodología apropiada & 7,45 & 1,81 \\
\hline El análisis de datos & 6,45 & 1,88 \\
\hline Revisión de los capítulos del TFG & 7,71 & 1,85 \\
\hline Nota final del TFG & 6,42 & 2,51 \\
\hline
\end{tabular}


Tabla 3.

Valoración del docente sobre la labor del tutor en la supervisión del TFG (continuación).

\begin{tabular}{|c|c|c|}
\hline Variable & Media & $\begin{array}{l}\text { Desviación } \\
\text { típica }\end{array}$ \\
\hline \multicolumn{3}{|l|}{$\begin{array}{l}\text { Grado de acuerdo/desacuerdo acerca del } \\
\text { papel del tutor (1 desacuerdo, } 5 \text { máximo } \\
\text { acuerdo): }\end{array}$} \\
\hline Debe motivar y animar al alumno & 3,91 & 1,18 \\
\hline Debe ser un experto en el tema del TFG & 3,17 & 0,91 \\
\hline $\begin{array}{l}\text { Debe dar apoyo académico en el desarrollo } \\
\text { del TFG }\end{array}$ & 3,34 & 1,14 \\
\hline Debe ayudar a escribir el TFG & 1,75 & 0,95 \\
\hline $\begin{array}{l}\text { Debe seleccionar el objeto y objetivos del } \\
\text { TFG }\end{array}$ & 2,17 & 1,25 \\
\hline Debe ayudar en la revisión de la literatura & 2,55 & 0.98 \\
\hline $\begin{array}{l}\text { Debe dar apoyo en la selección de la } \\
\text { metodología }\end{array}$ & 3,48 & 0,94 \\
\hline Es meramente un revisor del TFG & 1,68 & 1,10 \\
\hline $\begin{array}{l}\text { Debe comprometerse con el alumno a } \\
\text { responder a sus dudas o revisar el material } \\
\text { en un plazo determinado }\end{array}$ & 4,10 & 1,19 \\
\hline \multicolumn{3}{|c|}{$\begin{array}{l}\text { Valoración de las características clave que tiene que } \\
\text { tener un tutor (del } 1 \text { al } 10) \text { : }\end{array}$} \\
\hline Conocimiento de la materia & 6,86 & 2,18 \\
\hline Práctico y resolutivo & 7,82 & 1,67 \\
\hline Motivador & 7,58 & 2,27 \\
\hline Accesible y disponible & 7,82 & 1,73 \\
\hline Resolutivo ante los problemas & 7,62 & 1,87 \\
\hline Entusiasta e interesado en el tema & 7,27 & 1,96 \\
\hline Empático y de trato fácil & 7,41 & 1,72 \\
\hline Receptivo y dispuesto a ayudar al alumno & 7,82 & 1,92 \\
\hline Planificador & 7,13 & 1,82 \\
\hline Comunicador & 7,48 & 1,97 \\
\hline Paciente & 7,13 & 2,37 \\
\hline
\end{tabular}

Por otro lado, las características que debe tener un tutor se valoran en general de una manera muy similar: en torno a una puntuación de 7 sobre 10 , en las que se incluyen aspectos como ser práctico y resolutivo, ser entusiasta, receptivo, planificador y comunicador, aspectos destacados en trabajos previos (Derounian, 2011). Quizás el aspecto menos relevante a juicio de los propios docentes es el conocimiento de la materia, que obtiene la valoración más baja, en contra de los resultados obtenidos con anterioridad (Derounian, 2011). Resulta interesante, por lo tanto, que los propios directores señalen que lo importante no es ser experto del tema objeto de análisis, sino otras características y competencias que facilitan al alumno la realización del TFG.

\subsection{Uso de medios online en la dirección del TFG}

La encuesta recoge cuestiones relacionadas con la metodología de dirección del TFG y el uso de medios online. El principal resultado que podemos destacar de la tabla 4 en cuanto al uso de los medios online, es que el 91,67\% de los docentes encuestados los ha utilizado para la tutorización de los TFG, siendo el medio más frecuente el uso del email, cuyo uso asciende a 15 ocasiones en término medio al semestre, ligeramente superior al obtenido en otros estudios empíricos (Heinze \& Heinze, 2009; Del Río et al., 
2018). El resto de medios se utilizan significativamente menos, aunque se debe de destacar el uso de plataformas para compartir archivos (dropbox y semejantes), el uso de plataformas virtuales específicas de la universidad correspondiente y el uso creciente del Whatsapp, como había indicado Suárez-Lantarón (2017) en el ámbito de la tutoría.

Tabla 4.

Uso de medios online y aspectos organizativos de la supervisión del TFG.

\begin{tabular}{|c|c|c|}
\hline Variable & Media & $\begin{array}{l}\text { Desviación } \\
\text { típica }\end{array}$ \\
\hline Uso de medios online & $91,67 \%$ & ---- \\
\hline \multicolumn{3}{|l|}{ Tipo de medio online y frecuencia } \\
\hline Email & 15,93 & 21.21 \\
\hline Teléfono & 1,04 & 2.33 \\
\hline Skype & 0,66 & 1.92 \\
\hline WhatsApp & 2,25 & 8.31 \\
\hline Redes Sociales & 0,00 & 0,00 \\
\hline Plataforma virtual docente & 2,00 & 4,71 \\
\hline Dropbox y semejantes & 4,95 & 14,03 \\
\hline Otros & 0,12 & 0,61 \\
\hline \multicolumn{3}{|c|}{$\begin{array}{l}\text { Se ha llevado a cabo o no los siguientes } \\
\text { aspectos relacionados con las reuniones físicas }\end{array}$} \\
\hline $\begin{array}{l}\text { Planificar de antemano el número de } \\
\text { reuniones }\end{array}$ & $59,09 \%$ & -- \\
\hline $\begin{array}{l}\text { Planificar de antemano las fechas de las } \\
\text { reuniones }\end{array}$ & $54,55 \%$ & -- \\
\hline $\begin{array}{l}\text { Establecer a priori la duración de las } \\
\text { reuniones }\end{array}$ & $21,74 \%$ & -- \\
\hline $\begin{array}{l}\text { Establecer la siguiente reunión tras } \\
\text { terminar la última }\end{array}$ & $73,91 \%$ & -- \\
\hline $\begin{array}{l}\text { Exigir la entrega del material realizado } \\
\text { antes de la reunión }\end{array}$ & $73,91 \%$ & -- \\
\hline $\begin{array}{l}\text { Establecer un orden del día de la } \\
\text { reunión }\end{array}$ & $47,83 \%$ & -- \\
\hline $\begin{array}{l}\text { Dejar claro y por escrito los temas } \\
\text { tratados y los acuerdos de la última } \\
\text { reunión }\end{array}$ & $30,43 \%$ & -- \\
\hline $\begin{array}{l}\text { Establecer reuniones para grupos de } \\
\text { alumnos }\end{array}$ & $39,13 \%$ & -- \\
\hline \multicolumn{3}{|c|}{$\begin{array}{l}\text { En la supervisión del trabajo, grado de acuerdo y } \\
\text { desacuerdo en haber dejado claro... (1 desacuerdo, } 5 \\
\text { máximo acuerdo) }\end{array}$} \\
\hline $\begin{array}{l}\text { Las expectativas de tiempo que lleva el } \\
\text { TFG }\end{array}$ & 4,04 & 1,36 \\
\hline $\begin{array}{l}\text { Cuáles son las responsabilidades del } \\
\text { tutor y las del alumno }\end{array}$ & 3,82 & 1,33 \\
\hline Cómo se va a comunicar con el alumno & 4,26 & 1,09 \\
\hline $\begin{array}{l}\text { En cuanto tiempo va a responder al } \\
\text { alumno }\end{array}$ & 3,27 & 1,60 \\
\hline $\begin{array}{l}\text { Cómo se va a trabajar juntos } \\
\text { (planificación, reuniones...) }\end{array}$ & 4,21 & 0,95 \\
\hline
\end{tabular}

El uso de los medios online está positivamente correlacionado con el número de horas que cuenta un TFG en el Plan de Ordenación Docente (correlación 0,84), lo que viene a implicar que un mayor reconocimiento de la supervisión está asociado a un mayor uso de medios digitales.

En cuanto a los aspectos metodológicos, la tabla 4 detalla algunos resultados muy interesantes. En primer lugar, los docentes encuestados señalan que en la dirección del TFG afirman haber dejado claros los aspectos formales del mismo con el 
alumno, destacando especialmente el modo de comunicación con el alumno, aspecto esencial para una positiva relación académica entre tutor/director y alumno, y la planificación y establecimiento de reuniones. Estos resultados coinciden con el hecho de que una mayoría de docentes ha establecido previamente el número de reuniones a realizar y las fechas de éstas. Asimismo, en cuanto a las reuniones físicas, se ha especificado por parte del $75 \%$ de los docentes la exigencia de la entrega del material trabajado por parte del alumno antes de realizar la reunión con el tutor y el establecimiento de la siguiente reunión al finalizar la anterior. No obstante, son minoría los tutores que establecen a priori la duración de las reuniones y dejan por escrito lo tratado en cada reunión. Finalmente, destacar que sólo el $40 \%$ de los docentes realiza reuniones en grupo en la dirección de los TFG.

En relación con el apoyo institucional que se les da a los tutores del TFG por parte de la universidad, de manera generalizada, los docentes encuestados perciben que no se les valora adecuadamente las horas invertidas en la tutorización de los trabajos, confirmando indirectamente las afirmaciones de autores como Derounian, (2011) y Rowley y Slack, (2004), cuando afirman que esta actividad es intensiva en horas. Por otro lado, los tutores encuestados reconocen mayoritariamente que se les facilita información en cuanto a aspectos formales relacionados con plazos y requerimientos. Asimismo, señalan como aspecto mejorable el desarrollo de recursos y medios para facilitar la formación como tutor del TFG.

Finalmente, y resulta muy significativo y positivo, los docentes encuestados valoran muy positivamente, con una nota de 8 sobre 10, la relación mantenida con el alumno. Este resultado indudablemente es un aliciente para los docentes para seguir realizando su labor como director en futuros TFG. En la tabla 5 se recogen los estadísticos descriptivos de estas cuestiones.

Tabla 5.

Apoyo institucional y valoración de la relación con el alumno.

\begin{tabular}{|c|c|c|}
\hline \multirow[b]{2}{*}{$\begin{array}{l}\text { Variable } \\
\text { En relación al apoyo institucional, grado } \\
\text { acuerdo con las siguientes afirmaciones } \\
\text { (1 desacuerdo, } 5 \text { máximo acuerdo). La } \\
\text { Universidad/Facultad/Escuela.... }\end{array}$} & \multirow[t]{2}{*}{ Media } & \multirow[t]{2}{*}{ Desvia. típica } \\
\hline & & \\
\hline $\begin{array}{l}\text { Valoran adecuadamente las horas de } \\
\text { trabajo que dedica como tutor al TFG }\end{array}$ & 1,19 & 0,49 \\
\hline $\begin{array}{l}\text { Facilita información necesaria de plazos, } \\
\text { requerimientos, }\end{array}$ & 4,72 & 2,18 \\
\hline $\begin{array}{l}\text { Provee medios técnicos y tecnológicos } \\
\text { para las labores como tutor }\end{array}$ & 3,64 & 1,95 \\
\hline $\begin{array}{l}\text { Dedica recursos y medios para facilitar la } \\
\text { formación cómo tutor de TFG }\end{array}$ & 3,32 & 2,26 \\
\hline $\begin{array}{l}\text { Valoración relación mantenida con el } \\
\text { alumno (del } 1 \text { al 10) }\end{array}$ & 8,02 & 1,22 \\
\hline $\begin{array}{l}\text { Valoran adecuadamente las horas de } \\
\text { trabajo que dedica como tutor al TFG }\end{array}$ & 1,19 & 0,49 \\
\hline
\end{tabular}




\section{CONCLUSIONES}

Las conclusiones más importantes de este trabajo son las siguientes. En primer lugar, se observa un desfase muy significativo en la dirección de TFG entre el número de horas teóricas que se recogen en la programación docente de las universidades españolas y la dedicación real de los profesores. La dedicación teórica es de media de 9,5 horas por TFG, mientras que la dedicación real es de 3,6 horas/semana. Si tenemos en cuenta que la realización del TFG abarca un semestre, con 15 semanas hábiles, se está reconociendo únicamente el $18 \%$ de las horas reales que supone la dirección del TFG. Dado que se observa una relación positiva entre la implicación del profesor en la dirección del TFG y el reconocimiento del mismo, debería corregirse este desequilibrio con la finalidad de mejorar la implicación del docente.

En segundo lugar, en cuanto a las características de los tutores de TFG, los docentes encuestados destacan que el tutor debe especialmente motivar y animar al alumno, además de comprometerse a responderle en un plazo determinado. En general, como características personales, debe de ser práctico y resolutivo, entusiasta, receptivo, planificador y comunicador, siendo lo menos importante el conocimiento de la materia. De este modo, destacan por encima de todo, las habilidades de tipo relacional y de eficiencia de gestión. Por ello, resulta necesario plantear medidas de formación de los tutores para desarrollar este tipo de habilidades. Finalmente, se observa que la experiencia previa del tutor actúa de motivador del mismo en la dirección del TFG, además de ser más consciente de la importancia de fomentar la autonomía del alumno en su realización.

En tercer lugar, el análisis de las deficiencias más importantes que presentan los alumnos nos permite señalar algunas implicaciones necesarias. A partir de la principal deficiencia según los tutores, la redacción del TFG, se destaca la necesidad de realizar talleres específicos en este ámbito que mejoren las habilidades de redacción de los alumnos. Asimismo, los alumnos, en general, conocen los aspectos formales relacionados con el desarrollo del TFG, pero tienen menos claro en qué consiste realizarlo y cómo se realiza, presentando un bajo grado de autonomía y compromiso con el TFG. Por ello, los resultados indican la conveniencia de desarrollar algún tipo de formación básica sobre el mismo antes de iniciarse el proceso de dirección personal, en especial en lo que se refiere al establecimiento del tema y la selección de la metodología adecuada, que constituyen las etapas claves en el desarrollo del TFG, según el testimonio de los tutores encuestados. Finalmente, los profesores encuestados valoran muy positivamente la relación mantenida con el alumno, a pesar de estas problemáticas específicas.

En cuarto lugar, en cuanto al uso de medios online en la tutorización de TFG, es muy relevante que el $92 \%$ de los docentes haya utilizado herramientas online. El medio más usado es el email, destacando también plataformas para compartir archivos, plataformas docentes y el Whatsapp. De este modo, se pone de manifiesto la importancia de los medios online en la educación universitaria y, en particular, en la correcta supervisión de los TFG.

Lógicamente, el presente trabajo presenta algunas limitaciones, asociadas principalmente al tamaño de la muestra, que, si bien supera la de estudios anteriores en otros países, no se puede considerar representativa de los profesores universitarios que supervisan TFG en la universidad española. No obstante, el hecho de que la muestra abarque a docentes de nueve universidades públicas españolas de distintas áreas de conocimiento, dota de interés las percepciones de los mismos basadas en su amplia experiencia previa en la dirección de TFG. Futuros trabajos deberían ser más ambiciosos, abarcando muestras más grandes para que los resultados obtenidos puedan ser más concluyentes y que quizás más representativos. Asimismo, un análisis 
separado por áreas de conocimiento puede permitir testar si las características de esta supervisión varían por campos de conocimiento.

\section{REFERENCIAS}

Augustsson, G., \& Jaldemark, J. (2014). Online supervision: A theory of supervisors' strategic communicative influence on student dissertations. Higher Education, 67(1), 19-33. https://doi.org/10.1007/s10734-013-9638-4

Belletich Ruíz, O., \& Pérez de Villarreal, M.P. (2016). Elaboración de trabajos fin de grado. Superación de obstáculos y errores. Opcion, 32 (Special Issue 9), 218233.

Cook, M.C.F. (1980). The Role of the Academic Supervisor for Undergraduate Dissertations in Science and Science-Related Subjects. Studies in Higher Education, 5(2), 173-185. https://doi.org/10.1080/03075078012331377206

Da Cunha, I. (2016). El Trabajo de Fin de Grado y de Máster. Redacción, defensa y publicación. Barcelona: Editorial UOC.

Del Río, M.L., Díaz-Vázquez, R., \& Maside Sanfiz, J. M. (2018). Satisfaction with the supervision of undergraduate dissertations. Active Learning in Higher Education, 19(2), 159-172. https://doi.org/10.1177/1469787417721365

Derounian, J. (2011). Shall we dance? the importance of staff-student relationships to undergraduate dissertation preparation. Active Learning in Higher Education, 12(2), 91-100. https://doi.org/10.1177/1469787411402437

Expósito-Díaz, P., Freire-Esparis, M.P., Martínez-Roget, F., \& del Río-Araujo, M.L. (2018). El Trabajo de Fin de Grado: resultados desde una perspectiva de pares estudiantes/tutores. Revista de Docencia Universitaria, 16(2), 105-122. https://doi.org/10.4995/redu.2018.10190

Feather, D., Anchor, J. R., \& Cowton, C. J. (2014). Supervisors' perceptions of the value of the undergraduate dissertation. International Journal of Management Education, 12(1), 14-21. https://doi.org/10.1016/j.jme.2013.06.002

Greenbank, P., \& Penketh, C. (2009). Student autonomy and reflections on researching and writing the undergraduate dissertation. Journal of Further and Higher Education, 33(4), 463-472. https://doi.org/10.1080/03098770903272537

Greetham, B. (2009). How to write your undergraduate dissertation. Palgrave Macmillan. Hannigan, B., \& Burnard, P. (2001). Preparing and writing an undergraduate dissertation. Nurse Education in Practice, 1(4), 175-180. https://doi.org/10.1054/nepr.2001.0028

Heinze, A., \& Heinze, B. (2009). Blended e-learning skeleton of conversation: Improving formative assessment in undergraduate dissertation supervision. British Journal of Educational Technology, 40(2), 294-305. https://doi.org/10.1111/j.14678535.2008.00923.x

Jaldemark, J., \& Lindberg, J. O. (2013). Technology-mediated supervision of undergraduate students ' dissertations. Studies in Higher Education, (38), 13821392. https://doi.org/10.1080/03075079.2011.626851

Malcolm, M. (2012). Examining the implications of learner and supervisor perceptions of undergraduate dissertation research in Business and Management. Teaching in Higher Education, $17(5)$,

565-576. https://doi.org/10.1080/13562517.2011.641005 
Mills, C., \& Matthews, N. (2009). Review of tutor feedback during undergraduate dissertations: A case study. Journal of Hospitality, Leisure, Sport and Tourism Education, 8(1), 108-116. https://doi.org/10.3794/johlste.81.211

Ortiz-Repiso, V., García-Zorita, C., Pacios, A.R., \& Vianello, M. (2014). Planning and evaluating undergraduate dissertations: An experience at the Carlos III university bachelor's degree in information and documentation. Revista General de Informacion y Documentacion, 24(2), 278-304. h

Rekalde-Rodríguez, I. (2011). ¿Cómo afrontar el trabajo fin de grado? Un problema o una oportunidad para culminar con el desarrollo de las competencias. Revista Complutense de Educación, 22 (2), 179-193.

Roberts, L.D., \& Seaman, K. (2018). Good undergraduate dissertation supervision: perspectives of supervisors and dissertation coordinators. International Journal for Academic Development, 23(1), 28-40. https://doi.org/10.1080/1360144X.2017.1412971

Romero-Oliva, M. F. (Ed.) (2020). Escribir en la universidad: Elaboración y defensa de trabajos académicos -TFG, TFM-. Berna: Peter Lang.

Rowley, J. \& Slack, F. (2004). What is the future for undergraduate dissertations? Education + Training, 46(4), 176-181. https://doi.org/10.1108/00400910410543964

Shadforth, T., \& Harvey, B. (2003). The undergraduate dissertation: Subject-centred or student-centred? Electronic Journal of Business Research Methods, 2(2), 145152.

Suárez-Lantaron, B. (2017). WhatsApp como herramienta de apoyo a la tutoría. Revista de Docencia Universitaria, 15(2), 193-210. https://doi.org/10.4995/redu.2017.6941

Sussex, R. (2008). Technological options in supervising remote research students. Higher Education, 55(1), 121-137. https://doi.org/10.1007/s10734-006-9038-0

Starr-Glass, D., \& Ali, T. (2012). Double standards: When an undergraduate dissertation becomes the object of two different assessment approaches. Assessment and Evaluation in Higher Education, 37(2), 179-192. https://doi.org/10.1080/02602938.2010.515016

Todd, M., Bannister, P., \& Clegg, S. (2004). Independent inquiry and the undergraduate dissertation: Perceptions and experiences of final-year social science students. Assessment and Evaluation in Higher Education, 29(3), 335-355. https://doi.org/10.1080/0260293042000188285

Todd, M.J., Smith, K., \& Bannister, P. (2006). Supervising a social science undergraduate dissertation: Staff experiences and perceptions. Teaching in Higher Education, 11(2), 161-173. https://doi.org/10.1080/13562510500527693

Vera, J., \& Briones, E. (2015). Perspectiva del alumnado de los procesos de tutorización y evaluación de los trabajos de fin de grado. Cultura y Educacion, 27(4), 726765. https://doi.org/10.1080/11356405.2015.1089391

Webster, F., Pepper, D., \& Jenkins, A. (2000). Assessing the undergraduate dissertation. Assessment and Evaluation in Higher Education, 25(1), 71-80. https://doi.org/10.1080/02602930050025042 